EPJ Web of Conferences 92, 02114 (2015)

DOI: $10.1051 /$ epjconf/ 20159202114

(C) Owned by the authors, published by EDP Sciences, 2015

\title{
Effect of Moving Surface on NACA 63218 Aerodynamic Performance
}

\author{
Tayeb Yahiaoui ${ }^{1}$, Mohamed Belhenniche ${ }^{1}$ and Bachir Imine ${ }^{1, \mathrm{a}}$ \\ ${ }^{1}$ Laboratory of Aeronautics and Propulsive Systems, University of Sciences and Technology-MB, P.O 1505 \\ El-Mnaouer Oran Algeria
}

\begin{abstract}
The main subject of this work is the numerical study control of flow separation on a NACA 63218 airfoil by using moving surface. Different numerical cases are considered: the first one is the numerical simulation of non-modified airfoil NACA 63218 according at different angle of attack and the second one a set of moving cylinder is placed on leading edge of the airfoil. The rotational velocity of the cylinder is varied to establish the effect of momentum injection on modified airfoil aerodynamic performances. The turbulence is modeled by two equations k-epsilon model.
\end{abstract}

\section{Introduction}

The need for flow control emerged from the sixties years primarily to reduce wall friction, delay separation and reduce noise and vibration. To control the detachment of boundary layer, it became natural to intervene in the flow near the wall; because it is acting at the boundary layer it is possible to influence the aerodynamic characteristics of a global airfoil. Originally, the means of control were passive; they do not require an external energy supply. With the development of new technologies and improved knowledge about the internal dynamics of the turbulent boundary layer, control techniques available, passive or active, were extremely diverse. Since the publication of the first journals on the theory of boundary layer developed by Prandlt [1], a great challenge confronted scientists and engineers to minimize adverse effects and to further control flows.

Different methods such as synthetic jets, vortex generators, vortex manipulator were frequently made to experience this problem with a varied degree of success. A vast literature accumulated over the years in this area was actually reviewed by several authors as Ostowari [2], Tani [3], Chang [4] and others. However, the use of movable walls to control the boundary layer has received relatively less important. This technic called moving surface boundary-layer control is an active actuator to delay separation and control the flow of fluid around the body and improve the aerodynamic characteristics such as lift and drag. Among the most effective mechanisms for control by moving surface, we have to include the airfoils with rotating cylinder at the leading edge which is the subject of our present study. However, very few studies have focused on the complete characterization of the numerical and experimental mechanism and its influence on the flow. Similarly, the problems associated with the use of actuators have been little discussed. Mokhtarian [5] presented an experimental study of flow control using rotating cylinders of different shapes and various installation positions. A significant increase in maximum lift coefficient was recorded. A rotating cylinder at the leading edge of an airfoil seems to provide better results. Under optimal conditions, the lift coefficient greater than $200 \%$, and the separation was delayed until the angle $48^{\circ}$.

In the present work, a numerical investigation of flow around a modified NACA 63218 is presented. As shown in Figure 1, the modified airfoil is the cord $\mathrm{c}=150 \mathrm{~mm}$ with a rotating cylinder to the leading edge of $15 \mathrm{~mm}$ diameter (see the figure 2). Cylinder velocity Uc is 0,1 , 2,3 and 4 times of inlet velocity $U=20 \mathrm{~m} / \mathrm{s}$. The present study will be applied in the boundary layer which is the genesis of the phenomena governing the global dynamics of such flow control and aerodynamic performance of the modified airfoil.

\section{Transport equations and turbulence model}

The flow around the plane is considered turbulent asymmetrical. The general forms of the transport equations can be written in Cartesian coordinates as:

Continuity equation

$$
\frac{\partial}{\partial x_{j}}\left(\bar{\rho}_{\tilde{U}}\right)=0
$$

Momentum conservation equation

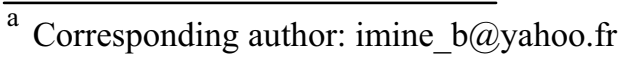




$$
\begin{gathered}
\frac{\partial}{\partial x_{j}}\left(\bar{\rho} \widetilde{U}_{i} \widetilde{U}_{j}\right)=\bar{\rho} g_{i}-\frac{\partial \bar{p}}{\partial x_{i}}-\frac{\partial}{\partial x_{j}}\left(\rho u_{i}{ }^{\prime \prime} u_{j}{ }^{\prime \prime}\right) \\
+\frac{\partial}{\partial x_{j}}\left[-\left(\frac{\partial \widetilde{U}_{i}}{\partial x_{j}}+\frac{\partial \widetilde{U}_{j}}{\partial x_{i}}\right)-\frac{2}{3} \bar{\mu} \frac{\partial \widetilde{U}_{k}}{\partial x_{k}} \delta_{i j}\right]
\end{gathered}
$$

The model of turbulence used in this present work is a model with two transport equations k-epsilon:

$$
\begin{aligned}
& \frac{\partial}{\partial t}(\bar{\rho} \tilde{k})+\frac{\partial}{\partial x_{i}}\left(\bar{\rho} \tilde{k} \tilde{u}_{i}\right)=\frac{\partial}{\partial x_{j}}\left[\left(\mu+\frac{\mu_{t}}{\sigma_{k}}\right) \frac{\partial \tilde{k}}{\partial x_{j}}\right] \\
& +G_{k}+G_{b}-\rho \varepsilon-Y_{M}+S_{k} \\
& \frac{\partial}{\partial t}(\rho \widetilde{\varepsilon})+\frac{\partial}{\partial x_{i}}\left(\rho \varepsilon \tilde{u}_{i}\right)=\frac{\partial}{\partial x_{j}}\left[\left(\mu+\frac{\mu_{t}}{\sigma_{k}}\right) \frac{\partial \widetilde{\varepsilon}}{\partial x_{j}}\right] \\
& +C_{1 \varepsilon} \frac{\tilde{\varepsilon}}{\tilde{k}}\left(G_{k}+C_{3 \varepsilon} G_{b}\right)-C_{2 \varepsilon} \rho \frac{\widetilde{\varepsilon}^{2}}{\widetilde{k}}+S_{\varepsilon}
\end{aligned}
$$

Production of turbulent kinetic energy $G_{k}$ is given by the formula:

$$
G_{k}=-\bar{\rho} \cdot \overline{u_{i}^{\prime \prime} \cdot u_{j}^{\prime \prime}} \frac{\partial \tilde{u}_{j}}{\partial x_{i}}
$$

The turbulent viscosity is obtained by:

$$
\mu_{t}=\bar{\rho} C_{\mu} \frac{\widetilde{k}^{2}}{\widetilde{\varepsilon}}
$$

The model constants are given in Table I:

Table 1. Constantes du modèle $k-\varepsilon$

\begin{tabular}{|c|c|c|c|c|}
\hline$C_{1 \varepsilon}$ & $C_{2 \varepsilon}$ & $C_{\mu}$ & $\sigma_{k}$ & $\sigma_{\varepsilon}$ \\
\hline 1,44 & 1,92 & 0,09 & 1,0 & 1,3 \\
\hline
\end{tabular}

(For more information see reference 3)

In the present work, the control of flow separation on a NACA 63218 airfoil by using moving surface is numerically investigated. Firstly the numerical simulation of non-controlled case is performed. According to existing model of NACA 63218 at different angle of attack and subsequently computational structured mesh are created in program Gambit.

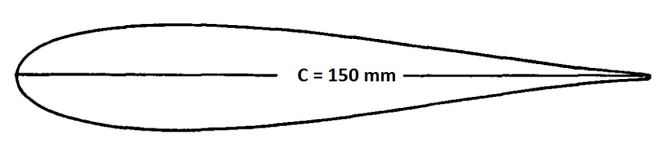

Figure 1. Baseline NACA 63218.

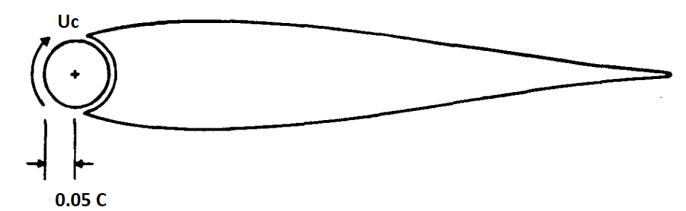

Figure 2. Modified airfoil NACA 63218.

\section{Meshing and boundary conditions}

For the airfoil case, NACA 63218 airfoil with $0.150 \mathrm{~m}$ of chord length $c$ and as it shown in figure 3 , the $3 \mathrm{D}$ structured mesh are generated. The upstream boundary of the computational domain is $3 c$ from the leading edge of the airfoil. The outflow boundary is located at $12 c$ downstream of the trailing edge. As it shown in figure 4, the three-dimensional and incompressible flow is solved in the case of modified airfoil. Turbulence is modeled by two equations k-epsilon model. Inlet boundary conditions are corresponding to the boundary conditions of the experiment. Second order upwind discretization scheme is selected with respect to the mesh used. The simulation is run acquired at $0^{\circ}$ through $32^{\circ}$ of incidence angle where stalling occurred. The Reynolds number based on the free stream velocity and the chord length is $2.13 * 10^{5}$. To achieve this Reynolds number, the velocity inlet is set at $20 \mathrm{~m} / \mathrm{s}$.

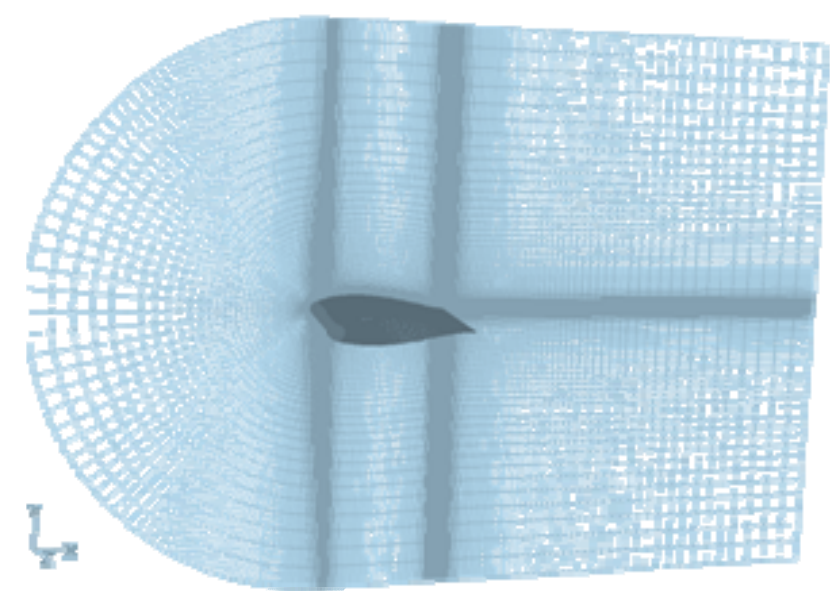

Figure 3. Meshing of calculation domain. 


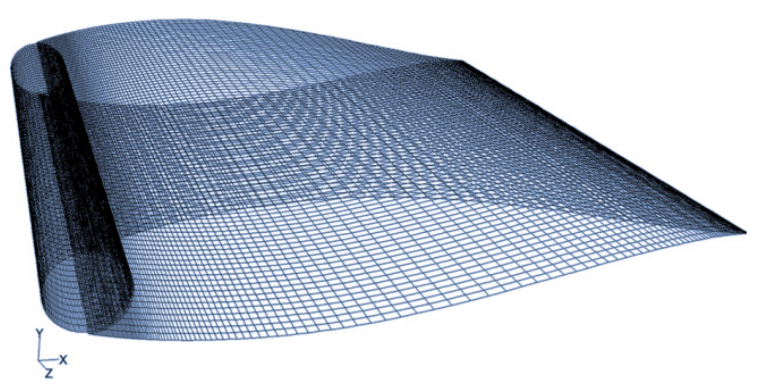

Figure 4 Meshing the modified airfoil.

As it shown in figure 3, typically the fluid box was modelled to have one inlet and one outlet and three slip surfaces, at the root of the geometry is being analysed. The geometry itself consisted of non-slip interior boundaries. The box also provided easy boundary specifications for the structural problems. Additionally, this method allowed easy estimation of the drag and lift forces produced by the various components, if desired.

\section{Results}

Several compilations were carried out for different angles of attack to determine the lift and drag. Each case was carried out at the same speed: 20 meters per second. The angle of attack extended from 0 to 30 degrees.

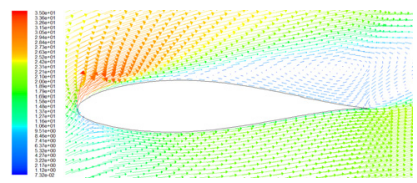

Baseline

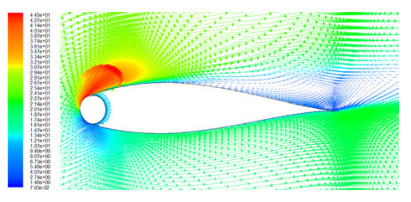

$\mathrm{Uc} / \mathrm{U}=1$

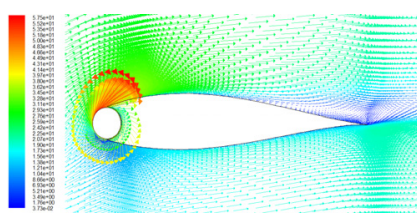

$\mathrm{Uc} / \mathrm{U}=3$

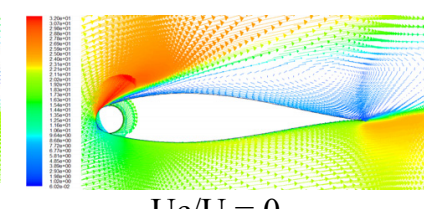

$\mathrm{Uc} / \mathrm{U}=0$

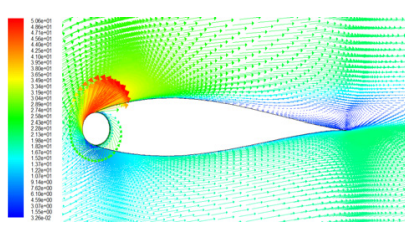

$\mathrm{Uc} / \mathrm{U}=2$

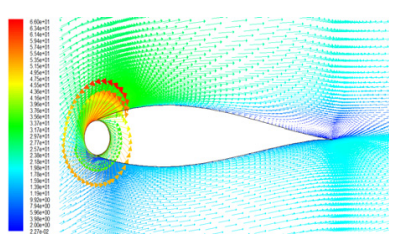

$\mathrm{Uc} / \mathrm{U}=4$
Figure 5. Computational visualizations of field vectors at $14^{\circ}$ incidence.

In the figure 5, the computational visualizations of field vectors showed quite clearly the effectiveness of this form of control of the boundary layer (rotating cylinder). In the case of $U c / U=0$, the cylinder has negatively affected the flow with the appearance of well-defined separation bulbs especially at high incidence angle, with large-scale vortices downstream of the airfoil than in baseline airfoil. However, with the rotating cylinder to Uc / $\mathrm{U}=1$, the flow becomes more stable and attached to the upper surface. $U c / U=2,3,4$, the flow in the major portion of the upper surface is glued to the airfoil. The detaching position of the boundary layer moves progressively with the increase in the velocity of the cylinder to the trailing edge then the stall has been delayed.

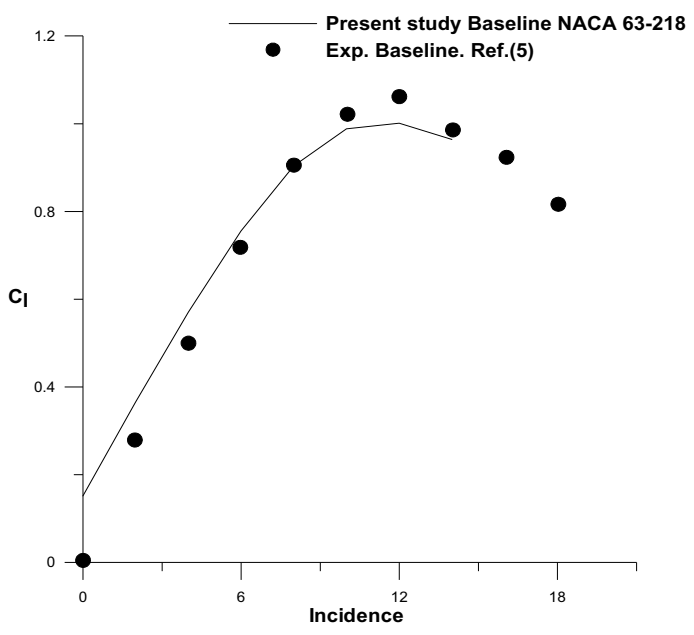

Figure 6. Evolution of the lift coefficient.

Figure 6 compares the computational evolution of the lift according to the angle of attack with the experimentally lift in the baseline case [5]. An agreement of the two curves is noted in the linear zone. However, examining the stall zone, the differences between the numerical study and deviations from the measured curve become visible. It is clear that the numerical simulation is far from predicting the stall, because the models currently used are not accurate for flows with separate streams. Based on this comparison, it was concluded that the limited use of CFD is the post-stall is to say, at angles of attack below $12^{\circ}$ or less.

In the figure 7 and in the case of establishment of a cylinder, different evolution of lift in the case of $U c / U=$ $0,1,2,3$ and 4 are shown. In the case of the absence of rotation of the cylinder to $\mathrm{Uc}=0 \mathrm{~m} / \mathrm{s}$, the gap created between the airfoil and the cylinder causes a decrease of the $\mathrm{C}_{\mathrm{Lmax}}=0.83$ corresponding to an incidence of $12^{\circ}$ and we note that the linear part remains practically unchanged. However, with the rotation of the cylinder, an injection flow is developed at the leading edge of the wing of causing a delay-ing off of the boundary layer. The stall occurs around the $28^{\circ}$ angle in the case of Uc / $\mathrm{U}=4$ with an increase of the lift coefficient of approximately $45 \%$. It remains to note that the increase in velocity of the cylinder beyond $U c / U=3$ is only slightly improves the situation suggested managing the existence of a critical velocity above which the rate added to the flow through the cylinder movement seems to have little effect. It is noted that the effect of the rotation of the cylinder is to extend the maximum lift without affecting the linear and without flattening the top of stall. 


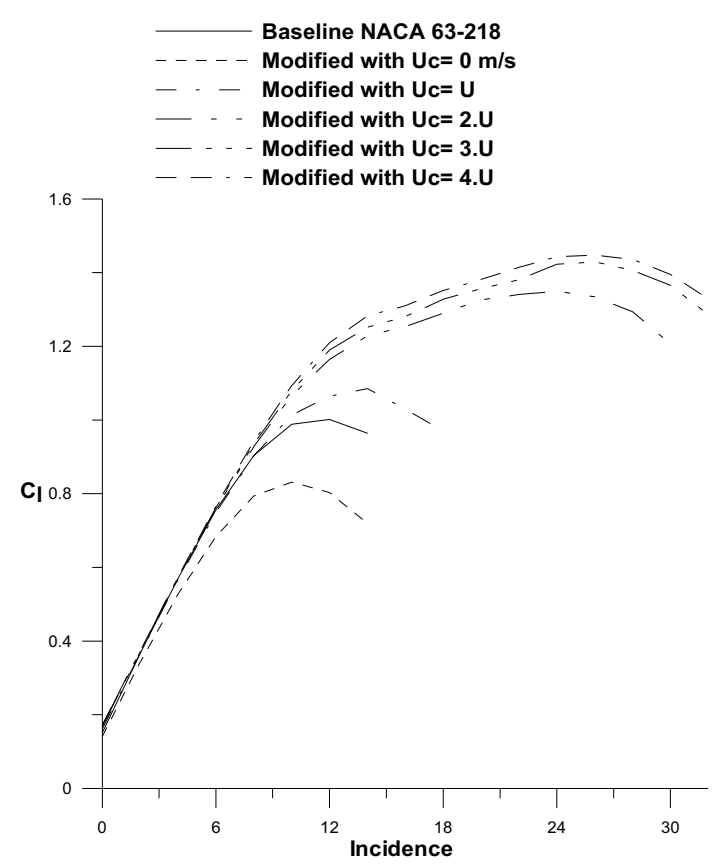

Figure7. Evolution of the lift coefficient

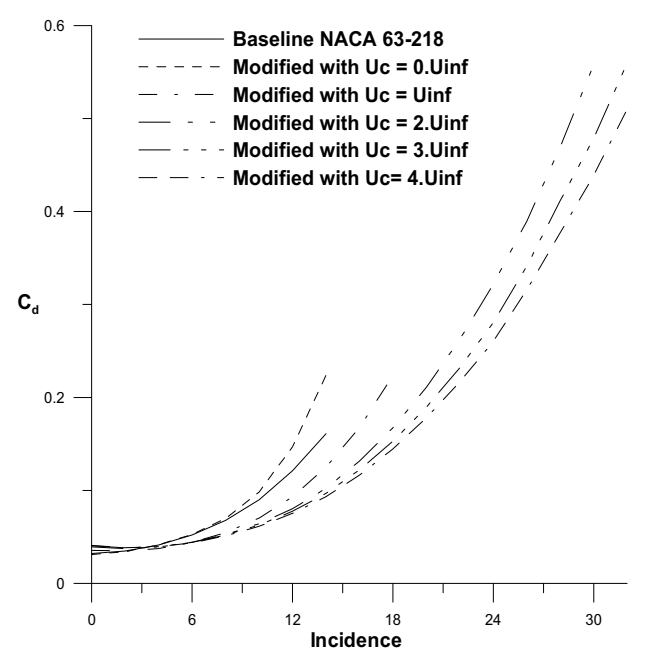

Figure 8. Evolution of the drag coefficient

The evolution of the drag coefficient according to the angle of attack is presented in figure 8 for different cases of $\mathrm{Uc} / \mathrm{U}=0,1,2,3$ and 4. All curves are parabolic type tendency similar to those found on the literature. In particular, one notes the increase in the drag with the increase in the angle of attack.. In the case of implementation of the cylinder, an increase of the drag in the case of $U c / U=0$ is observed which is due to the presence of the gaps between the stationary cylinder and the airfoil. From $U c / U=1$, the trail has experienced a significant reduction: for angle of incidence of $14^{\circ}$, the drag coefficient reached the value of 0.25 for the case of $\mathrm{Uc} / \mathrm{U}=0$, and that it did not exceed the value of $0.1 \mathrm{Uc}$ $/ \mathrm{U}=1$.In addition, and from $\mathrm{Uc} / \mathrm{U}=2$, we note that the rotation of the cylinder seems to provide less drag reduction effect for the same term as the coefficient of lift.

\section{Conclusion}

The results of this numerical investigation showed several benefits of the moving surface compared with a conventional airfoil. Also, the results showed that as the angular momentum of the moving surface is increased, the overall lift force increased and the drag force decrease. In addition to increasing lift, moving surface inhibit stall at high angles of attack. The most intriguing result of this research is the capability for moving surface aircraft to perform short take-offs and landings.

\section{References}

1. S. M. Lam, The Prandtl Boundary Layer Theory. ME351B Fluid Mechanics, Stanford University (2003)

2. C. Ostowari, D. Naik, Post stall studies of untwisted varying aspect ratio blades with an NACA4415 airfoil section part 1. Wind Engineering, 8, 176 (1984)

3. I. Tani, Boundary-Layer Transition, Annual Review of Fluid Mechanics, 1, 169 (1969)

4. R. D. Joslin, C. L. Streett, I. Chang, Spatial Direct Numerical Simulation of Boundary-Layer Transition Mechanisms: Validation of PSE Theory Theor. \& Comput.. Fluid Dyn, (1993)

5. F. Mokhtarian, Fluid dynamics of Airfoils With Moving Surface Boundary Layer Control, PHD thesis of the University of British Colombia (1988) 